\title{
Diagnostic Efficacy of Vessel Specific Coronary Calcium Score in Detection of Coronary Artery Stenosis
}

\author{
Marzieh Motevalli ${ }^{1}$; Hossein Ghanaati ${ }^{2}$; Kavous Firouznia ${ }^{2}$; Jalal Kargar ${ }^{3}$; Mounes Aliyari \\ Ghasabeh $^{4, *}$; Mona Shahriari ${ }^{4}$; Amir Hosein Jalali ${ }^{4}$; Madjid Shakiba ${ }^{4}$ \\ ${ }_{1}^{1}$ Department of Radiology, Shahid Rajaie Cardiovascular, Medical and Research Center, Iran University of Medical Sciences, Tehran, IR Iran \\ ${ }_{2}^{2}$ Department of Radiology, Advanced Diagnostic and Interventional Radiology Research Center (ADIR), Tehran University of Medical Sciences, Tehran, IR Iran \\ ${ }_{3}^{3}$ Department of Radiology, Imam Khomeini Hospital, Tehran University of Medical Sciences, Tehran, IR Iran \\ ${ }^{4}$ Advanced Diagnostic and Interventional Radiology Research Center(ADIR), Tehran University of Medical Sciences, Tehran, IR Iran \\ ${ }^{*}$ Corresponding Author: Mounes Aliyari Ghasabeh, Advanced Diagnostic and Interventional Radiology Research Center (ADIR), Medical Imaging Center, Imam Khomeini Hospital, \\ Keshavarz Blvd., Tehran, Iran. Tel: +98-2166581579, Fax: +98-2166581578, E-mail: mnaliyari@gmail.com
}

Received: December 11, 2014; Accepted: December 19, 2014

Background: Coronary artery calcification which is determined quantitatively by coronary calcium scoring has been known as a sign of coronary stenosis and thus future cardiac events; hence it has been noticed on spotlight of researchers in recent years. Developing different method for early and optimal detection of coronary artery disease (CAD) is really essential as CAD are the first cause of death in population.

Objectives: To evaluate predictive value of vessel specific coronary artery calcium (CAC) score in predicting obstructive coronary artery disease.

Patients and Methods: In this diagnostic test study we evaluated patients with coronary computed tomography angiography (CCTA) and CAC score which had been referred to two referral radiology center in Tehran, Iran and finally we selected 2525 patients in a single and sequential pattern to create a diagnostic study. The whole-heart CAC scores and vessel specific CAC scores were calculated individually for the 4 major epicardial coronary arteries in 2 distinct group; group A ( patients with previous history of CABG) and group B (patients without history of $\mathrm{CABG}$ ). For evaluation of obstruction tree cut off points were described: $0>$; at least 1 segment with any kind of stenosis, $\geq 50$; at least 1 segment with stenosis $\geq 50, \geq 70$; at least 1 segment with stenosis $\geq 70$.

Results: Mean of coronary calcium scores in terms of each coronary artery vessel increase by increasing coronary stenosis grade in group B; LAD, RCA, LCX respectively have mean CAC score 6.06,6.21 and 5.04 in normal patients and 221.6,226.7 and 106.6 in patients with complete stenosis. As expected these findings don't work for group A. Also By increasing calcium score cutoff in all four vessels sensitivity decreased and specificity increased but steal LAD had higher sensitivity than other vessels and LM had higher specificity. Thus using calcium score method is useful for ruling out stenosis in LAD while calcium score of LM can predict existence of stenosis in LM. However none of the vessel specific CAC can reach to $100 \%$ sensitivity and specificity of CCTA method.

Conclusions:CCTA is highly superior than vessel specific CAC score thus to minimize patients radiation does maybe we can eliminate CAC scan as a routinely perform procedure at the beginning of the CCTA.

Keywords: Computed Tomography; Coronary Angiography; Coronary Artery Disease

\section{Background}

According to American Heart Association (AHA); coronary artery disease (CAD) is the most common cause of death in population over 20 years of age (1) and it almost costs the United States \$108.9 billion each year (2) thus it is comprehensible why obtaining different methods and developing various technologies and grading systems to determine risk of CAD and detecting early stage of the disease is very important in this field. CT scan has emerged as an important modality in the diagnosis of cardiothoracic pathologies (3-7). In recent years, rapid development of coronary computed tomography angiography (CCTA) technology leads to increase diagnostic accuracy and predictive value of CCTA in detecting CAD. As a noninvasive procedure, CCTA is a desirable method for either patients or physicians and many researches has been focused on determining CCTA diagnostic accuracy compared with invasive coronary angiography (ICA) as the gold standard. Different studies have been shown that 64-slice multi detector CT scan that is the most usual scanner using worldwide for CAD detection has $90-94 \%$ sensitivity and 96-100\% negative predictive value (NPV) in diagnosis of coronary stenosis (8-10) and more advanced devices as 128- slice multi detector CT scan has higher detection rate (11). On the other hand, coronary artery calcification which is determined quantitatively by coronary calcium scoring has been known as a sign of coronary stenosis and thus future cardiac events; hence it has been also noticed on spotlight of researchers in recent years

Copyright (C) 2014, Iranian Red Crescent Medical Journal. This is an open-access article distributed under the terms of the Creative Commons Attribution-NonCommercial 4.0 International License (http://creativecommons.org/licenses/by-nc/4.0/) which permits copy and redistribute the material just in noncommercial usages, provided the original work is properly cited. 
(12). Coronary calcium scan is a fairly simple test that is routinely performed at the beginning of the CCTA, and its radiation dose use for calcium scanis less than conventional CCTA $(13,14)$. As coronary calcium score (CCS) could be considered as an accurate sign of coronary stenosis, it could be considered as a good predictor of future cardiac events beside other cardiac risk assessment systems (15, 16). In addition, it can predict existent coronary stenosis in patientcandidate for CCTA thus finding new methods to improve CCS accuracy in coronary artery obstruction diagnosing is absolutely needed.

\section{Objectives}

The purpose of this study was evaluating coronary artery calcium scoring predictive value in detecting or ruling out stenosis by vessel specific CAC score method in patients suspected of CAD and comparing these values with CCTA findings.

\section{Patients and Methods}

\subsection{Study Design and Patients' Enrollment}

In this cross sectional, diagnostic test study performed on patients referred to two referral multislice CT centers in Tehran, Iran between January 2008 and May 2013. Totally 2770 consecutive stable patients with signs and symptoms suggestive of CAD or follow up of post coronary artery bypass graft (CABG) underwent cardiac multi slice CT scan. The patients were sequentially enrolled in the study. Among them, 245 patients with the history of previous coronary artery stent placement were excluded from study because artifact due to stent presence in CT imaging that could interfere in cardiac calcium scoring. The remaining 2525 patients divided in to two groups. One group patients with previous history of CABG. Other group includes patients without history of CABG. After obtaining written informed consent from patients, all patients underwent CCTA and calcium scoring. Study design approve by Institutional review committee of centers. In all patients, individual calcium scoring of main coronary arteries were determined. In addition, degree of stenosis in each main coronary arteries were determined in CT. Then the accuracy of calcium scoring for diagnosis of significant coronary stenosis was determined.

\subsection{CT Protocol and Image Acquisition}

All patients were scanned on a dual source, 128- slices multi detector CT scan (Somatom Definition Flash, Siemens Medical Solutions, Syngo CT2011A, Germany). patients that were on $\beta$-blocker continued their medication but other patients didn't receive $\beta$-blocker to decrease heart rate (the 128-slice dual source scanner is a very fast scanner that can provide high quality images in a patient with high or irregular heart rate and it's not necessary to prescribe a $\beta$-blocker before scan to control heart rate
(17). Before coronary CT angiography a non-enhanced prospective ECGgated scan was performed for CAC scoring with the following parameters: detector collimation $32 \times 0.6 \mathrm{~mm}$; slice acquisition $128 \times 0.6 \mathrm{~mm}$; gantry rotation time $280 \mathrm{~ms}$; pitch of 0.2-0.35 adapted to the heart rate, tube current- time $400 \mathrm{mAs}$ per rotation; and tube voltage $120 \mathrm{kV}$. Following calcium scoring, all patients received a single puff of Nitroglycerin $0.4 \mathrm{mg}$ (GLYTRIN $0.4 \mathrm{mg} /$ dose, sanofi, MoretonWirral, UK). After 2 minutes, a bolus of $80 \mathrm{~mL}$ of Iohexol (Omniopaque, $350 \mathrm{mg} \mathrm{I} / \mathrm{mL}$, GE Healthcare, Ireland cork, Ireland) followed by $30 \mathrm{~mL}$ saline chaser, was injected into an antecubital vein via an 19-gauge catheter (injection rate $6 \mathrm{~mL} / \mathrm{s}$ ) (18). Contrastagent application was controlled by bolus-tracking in the aortic root. CT angiography and Image acquisition started 10 seconds after the signal attenuation reached the predefined threshold of 130 Hounsfield units (HU). ECG-pulsing for radiation dose reduction was applied in all patients. With two tubes and two detectors mounted at orthogonal orientation in the gantry, the transmission data required for the reconstruction of one image slab can be acquired in 3-5 seconds breath holding. Axial images were reconstructed with $3 \mathrm{~mm}$ slice thickness and $0.3 \mathrm{~mm}$ increment using a medium sharp convolution kernel (B20) and retrospective ECG gating. The reconstructions were performed in best of diastole and systole for using circulation application.

\subsection{Image Analysis}

For image analysis coronary artery tree was separated to 18 segments (within 4 main coronary artery vessels: LM, Left main; LAD, left anterior descending artery; RCA, right coronary artery; LCX, left circumflex artery) according to Society of Cardiovascular Computed Tomography (SCCT) Guidelines (19); each segment diameter was > 1.5 $\mathrm{mm}$. for assessment of stenosis severity we used Recommended Quantitative Stenosis Grading with a little modification. Recommended Quantitative Stenosis Grading defines coronary stenosis severity as: o Normal: Absence of plaque and noluminal stenosis. 1 Minimal: Plaque with <25\% stenosis. 2 Mild: 25\%-49\% stenosis. 3 Moderate: 50\%69\% stenosis. 4 Severe: 70\%-99\% stenosis. 5 Occluded. We modified this grading to: 0 Normal: Absence of plaque and no luminal stenosis. 1 Mild: $1 \%-49 \%$ stenosis. 2 Moderate: $50 \%-69 \%$ stenosis. 3 Severe: $70 \%-99 \%$ stenosis. 4 Occluded: $100 \%$ stenosis. In addition for evaluation of obstruction in each of the 4 coronary artery, tree cut off points were described: $0>$; at least 1 segment with any kind of stenosis, $\geq 50$; at least 1 segment with stenosis $\geq 50$, $\geq$ 70 ; at least 1 segment with stenosis $\geq 70$. Coronary artery calcium (CAC) scores which identify as distribution of imaging pixels with calcification density $>130 \mathrm{HU}$ were calculated in each coronary artery (LM, LAD, RCA, LCX) by using Agatston score method, total coronary artery calcium score were also calculated, reference norms for calcium score and Agatstone's scoring system describe in Table 1 (20). The whole cardiac CAC scores and vessel specific CAC 
Motevalli M et al.

scores were calculated separately. Each patient coronary CT angiogram was evaluated by two experience cardio thoracic interventional radiologist that were unaware of clinical history of patients. Consensus were obtain by consultation on cases with different report.

\subsection{Statistical Analysis}

The statistical analysis of the collected data was performed by SPSS version 18. We checked in all variables the normality with kolmogorov-smirnov test. If the data showed normal distribution, we used parametric test and if the data did not show normal distribution, we used nonparametric equivalent test instead. At first one way ANOVA or equivalent nonparametric kruskal wallis test was performed to compare mean CCS value in different stenosis severity groups of stenosis in each coronary arteries [normal, mild stenosis, moderate stenosis, severe stenosis]. Then ROC curves and the area under the curve (AUC) were used to evaluate the diagnostic efficacy of different CCS parameters in different cut of point in diagnosing coronary stenosis [any stenosis, more than 50\% stenosis, more than $70 \%$ stenosis] $\mathrm{P}$ values of less than 0.05 were considered to indicate statistical significance. 95\% confidence intervals are also presented.

\section{Results}

\subsection{Study Population}

Totally 2525 patients were enrolled in this study; 1562 were male (59.7\%) and the mean age of patients was 57.1 \pm 12.5 years [7-93]. Totally, 506 patients had history of previous CABG surgery and evaluated as a separate group (group A), the other 2019 remaining patients had not history of CABG (group B). Both groups underwent coronary artery calcium scoring and CCTA.

\subsection{Results of CAC Scoring and Coronary CT Angi- ography}

\subsubsection{The Whole Cardiac CAC Scores}

Total CAC scoring were interpreted according to agatston's scoring system and included: In the group With history of CABG (Group A): 23 patients (4.5\%); no identifiable calcification, 13 patients (2.5\%); minimal calcification, 38 patients (7.4\%); mild calcification, 128 patients (25\%); moderate calcification, 304 patients ( 59.9\%); significant calcification. In the group without history of proven CAD (group B): 1017 patients (50.3\%); no identifiable calcification, 157 patients (7.7\%); minimal calcification, 332 patients (16.4\%); mild calcification, 252 patients (12.4\%); moderate calcification, 261 patients (12.9\%); significant calcification. CABG group mostly include patients with high rate of calcification and in contrast in the group without history of CABG aggregation of patients with low rate of calcification is high. According to patients distribution in each group this finding had expected.

\subsubsection{Total CCTA Findings According to Tree cut of Points}

Group A: 4 patients (0.79\%) had completely normal coronary arteries, 502 patients (99.2\%) had at least one segment with any kind of stenosis, 502 patients (99.2\%) had at least one segment with stenosis $\geq 50 \%$ and 498 patients (98.2\%) had at least one segment with stenosis $\geq 70 \%$.Group B: 943 patients (46.7\%) had completely normal coronary arteries, 1076 patients (53.2\%) were detected with any kind of stenosis, 727 patients (36\%) had at least one segment with stenosis $\geq 50 \%$ and 446 patients (22.7\%) had at least one segment with stenosis $\geq 70 \%$.

\subsection{Vessel Specific Coronary Artery Stenosis and CAC Score}

Vessel specific coronary artery stenosis distribution according to Quantitative Stenosis Grading system (Normal, Mild, Moderate, Severe and Occluded stenosis) and mean of calcium scoring in each subset were described in details in Table 2. Higher grade of coronary artery obstruction were associated with a greater extent of CAC score [All of p-values lower than 0.05 in each coronary arteries] but it's not reliable for completely obstructed artery and coronary artery with the previous CABG surgery.

\subsection{Diagnostic characteristics of Group $A$ and Group B CAC Scores by ROC Analysis}

ROC curves were constructed and AUC were calculated to distinguish vessel specific CCS correlation with vessel specific CCTA findings. Overall, CAC score in patients without previous history of CABG had higher predictive value than group A. In group A, In particular, CAC score in RCA had higher correlation with coronary artery stenosis grade than other coronary arteries. In group B, CAC scoring had almost similar correlation with coronary artery stenosis grade in LAD, RCA and LCX, although CAC scoring in LM wasn't reliable. Corresponding optimal calcium scores cutoffs with corresponding sensitivity, specificity,

\begin{tabular}{lcccc}
\hline Table 1. Reference Norms for Calcium Score & & \\
\hline $\begin{array}{l}\text { No Identifiable } \\
\text { Calcification }\end{array}$ & $\begin{array}{c}\text { Minimal No Identifiable } \\
\text { Calcification }\end{array}$ & Mild Calcification & Moderate Calcification & Significant Calcification \\
\hline $\mathbf{0}$ & $1-10$ & $11-100$ & $101-400$ & 401 and above \\
\hline
\end{tabular}


Motevalli M et al.

PPV, NPV, and test accuracy are shown in Table 3. In group B in $\geq 50 \%$ stenosis, CAC score method showed higher AUC in LAD than other vessels (LAD CACs AUC: $85 \%$; RCA CACs AUC: 83\%; LCX CACs AUC: 82\%; LM CACs AUC: 69\%). A similar pattern was also seen in $\geq 70 \%$ stenosis. In group A the performance of CACs decreased (lower AUC values) and the pattern of AUC differed. In low CACs (cutoff 1) LAD had higher sensitivity than other vessels in both group (LM had the lower sensitivity) and sensitivity improved in $\geq$
$70 \%$ stenosis compared with $\geq 50 \%$ stenosis but specificity in both group and both category of stenosis in LAD is the least among 4 coronary arteries (LM had the highest specificity). By increasing calcium score cutoff in vessel sensitivity decreased and specificity increased but steal LAD had higher sensitivity than other vessels and LM had higher specificity. Thus using calcium score method is useful for ruling out stenosis in LAD while calcium score of LM can predict existence of stenosis in LM (Figures 1 - 4).

\begin{tabular}{|c|c|c|c|c|}
\hline & \multicolumn{2}{|c|}{ Without History of CABG } & \multicolumn{2}{|c|}{ With History of CABG } \\
\hline & Number & Mean \pm SD & Number & Mean \pm SD \\
\hline \multicolumn{5}{|c|}{ Normal } \\
\hline LM & 1804 & $1.48 \pm 15.706$ & 283 & $11.90 \pm 48.50$ \\
\hline LAD & 1046 & $6.06 \pm 29.55$ & 11 & $721.3 \pm 1040.1$ \\
\hline RCA & 1453 & $6.21 \pm 58.84$ & 49 & $148.4 \pm 495.3$ \\
\hline LCX & 1522 & $5.042 \pm 26.04$ & 98 & $122.5 \pm 427.9$ \\
\hline \multicolumn{5}{|c|}{ Mild [1-50] } \\
\hline LM & 153 & $18.50 \pm 47.39$ & 109 & $13.53 \pm 44.8$ \\
\hline LAD & 324 & $80.54 \pm 284.28$ & 5 & $150.1 \pm 132.40$ \\
\hline RCA & 226 & $80.98 \pm 256.37$ & 40 & $151.2 \pm 267.8$ \\
\hline LCX & 219 & $47.4 \pm 104.9$ & 46 & $86.5 \pm 162.01$ \\
\hline \multicolumn{5}{|c|}{ Moderate [51-70] } \\
\hline LM & 28 & $27.18 \pm 45.85$ & 37 & $20.26 \pm 73.34$ \\
\hline LAD & 201 & $129.7 \pm 224.5$ & 13 & $101.4 \pm 179.7$ \\
\hline RCA & 72 & $179.3 \pm 269.6$ & 27 & $158.6 \pm 213.28$ \\
\hline LCX & 88 & $99.8 \pm 136.2$ & 51 & $109.9 \pm 117.9$ \\
\hline \multicolumn{5}{|c|}{ Severe [70-99] } \\
\hline LM & 34 & $50.48 \pm 90.72$ & 71 & $49.86 \pm 94.74$ \\
\hline LAD & 280 & $256.2 \pm 308.11$ & 247 & $374.4 \pm 385.59$ \\
\hline RCA & 169 & $272.5 \pm 526.7$ & 176 & $321.4 \pm 472.3$ \\
\hline LCX & 146 & $105.0 \pm 160.12$ & 193 & $149.7 \pm 262.5$ \\
\hline \multicolumn{5}{|c|}{ Complete [100] } \\
\hline LM & 0 & 0 & 6 & $21.06 \pm 48.53$ \\
\hline LAD & 168 & $221.6 \pm 285.55$ & 230 & $397.2 \pm 471.1$ \\
\hline RCA & 99 & $226.7 \pm 407.3$ & 214 & $217.7 \pm 249.5$ \\
\hline LCX & 44 & $106.6 \pm 141.99$ & 118 & $146.2 \pm 202.9$ \\
\hline \multicolumn{5}{|l|}{ Total } \\
\hline LM & 2019 & $3.95 \pm 24.85$ & 506 & $18.30 \pm 59.68$ \\
\hline LAD & 2019 & $82.97 \pm 217.51$ & 506 & $383.1 \pm 447.8$ \\
\hline RCA & 2019 & $53.8 \pm 226.4$ & 506 & $238.6 \pm 372.9$ \\
\hline LCX & 2019 & $23.21 \pm 77.3$ & 506 & $133.8 \pm 273.9$ \\
\hline
\end{tabular}

a Abbreviations: CAD, coronary artery disease; LM, left main; LAD, left anterior descending artery; RCA, right coronary artery; LCX, left circumflex artery.

Table 3. ROC Curve Analysis a,b

\begin{tabular}{|c|c|c|c|c|c|c|c|}
\hline & AUC & Cutoff & Sensitivity, \% & Specificity,\% & PPV, \% & NPV, \% & Accuracy, \% \\
\hline \multicolumn{8}{|c|}{ Without history of CABG } \\
\hline \multicolumn{8}{|c|}{ Any stenosis } \\
\hline LM & 0.63 & 40 & 0.17 & 0.99 & 0.65 & 0.90 & 0.90 \\
\hline LAD & 0.84 & 22 & 0.64 & 0.94 & 0.90 & 0.73 & 0.79 \\
\hline RCA & 0.82 & 10 & 0.62 & 0.94 & 0.79 & 0.86 & 0.84 \\
\hline LCX & 0.79 & 23 & 0.47 & 0.95 & 0.75 & 0.84 & 0.83 \\
\hline \multicolumn{8}{|c|}{$>50 \%$ stenosis } \\
\hline \multirow[t]{3}{*}{ LM } & 0.69 & 1 & 0.43 & 0.94 & 0.16 & 0.97 & 0.92 \\
\hline & & 42 & 0.27 & 0.99 & 0.44 & 0.97 & 0.96 \\
\hline & & 102 & 0.14 & 0.10 & & 0.97 & 0.97 \\
\hline
\end{tabular}


Motevalli M et al.

\begin{tabular}{|c|c|c|c|c|c|c|c|}
\hline \multirow[t]{3}{*}{$\mathrm{LAD}$} & 0.85 & 1 & 0.85 & 0.75 & 0.61 & 0.91 & 0.78 \\
\hline & & 22 & 0.74 & 0.85 & 0.69 & 0.87 & 0.81 \\
\hline & & 10 & 0.80 & 0.81 & 0.66 & 0.89 & 0.80 \\
\hline \multirow[t]{3}{*}{$\mathrm{RCA}$} & 0.83 & 1 & 0.77 & 0.82 & 0.46 & 0.94 & 0.81 \\
\hline & & 11 & 0.70 & 0.88 & 0.54 & 0.93 & 0.84 \\
\hline & & 27 & 0.62 & 0.92 & 0.60 & 0.92 & 0.86 \\
\hline \multirow[t]{3}{*}{ LCX } & 0.82 & 1 & 0.76 & 0.82 & 0.40 & 0.95 & 0.81 \\
\hline & & 23 & 0.57 & 0.92 & 0.53 & 0.93 & 0.87 \\
\hline & & 10 & 0.65 & 0.89 & 0.48 & 0.94 & 0.85 \\
\hline \multicolumn{8}{|c|}{$>70 \%$ stenosis } \\
\hline \multirow[t]{3}{*}{ LM } & 0.64 & 1 & 0.35 & 0.94 & 0.84 & 0.98 & 0.92 \\
\hline & & 57 & 0.29 & 0.99 & 0.31 & 0.98 & 0.97 \\
\hline & & 156 & 0.17 & 0.10 & & 0.98 & 0.98 \\
\hline \multirow[t]{3}{*}{$\mathrm{LAD}$} & 0.84 & 1 & 0.88 & 0.65 & 0.34 & 0.96 & 0.68 \\
\hline & & 22 & 0.81 & 0.76 & 0.41 & 0.94 & 0.76 \\
\hline & & 11 & 0.84 & 0.72 & 0.39 & 0.95 & 0.74 \\
\hline \multirow[t]{3}{*}{ RCA } & 0.82 & 1 & 0.77 & 0.79 & 0.32 & 0.96 & 0.78 \\
\hline & & 14 & 0.72 & 0.86 & 0.40 & 0.95 & 0.84 \\
\hline & & 27 & 0.65 & 0.89 & 0.44 & 0.94 & 0.86 \\
\hline \multirow[t]{3}{*}{ LCX } & 0.81 & 1 & 0.78 & 0.79 & 0.25 & 0.97 & 0.78 \\
\hline & & 23 & 0.57 & 0.89 & 0.32 & 0.95 & 0.86 \\
\hline & & 10 & 0.65 & 0.86 & 0.30 & 0.96 & 0.84 \\
\hline \multicolumn{8}{|c|}{ With history of CABG } \\
\hline \multicolumn{8}{|c|}{ any stenosis } \\
\hline LM & 0.55 & 120 & 0.8 & 0.98 & 0.73 & 0.57 & 0.58 \\
\hline LAD & 0.39 & 46 & 0.84 & 0.10 & 0.97 & 0.01 & 0.82 \\
\hline RCA & 0.75 & 56 & 0.62 & 0.74 & 0.95 & 0.17 & 0.63 \\
\hline LCX & 0.63 & 26 & 0.67 & 0.55 & 0.85 & 0.28 & 0.64 \\
\hline \multicolumn{8}{|c|}{$>50 \%$ stenosis } \\
\hline \multirow[t]{3}{*}{ LM } & 0.51 & 1.1 & 0.33 & 0.82 & 0.34 & 0.80 & 0.70 \\
\hline & & 25 & 0.25 & 0.88 & 0.36 & 0.80 & 0.73 \\
\hline & & 149 & 0.10 & 0.99 & 0.73 & 0.79 & 0.78 \\
\hline \multirow[t]{3}{*}{ LAD } & 0.48 & 1 & 0.93 & 0.70 & 0.96 & 0.02 & 0.90 \\
\hline & & 45 & 0.84 & 0.13 & 0.96 & 0.02 & 0.81 \\
\hline & & 126 & 70 & 19 & 0.96 & 0.02 & 0.68 \\
\hline \multirow[t]{3}{*}{ RCA } & 0.70 & 1 & 0.89 & 0.40 & 0.85 & 0.25 & 0.69 \\
\hline & & 56 & 0.65 & 0.70 & 0.90 & 0.29 & 0.65 \\
\hline & & 20 & 0.77 & 0.50 & 0.87 & 0.31 & 0.72 \\
\hline \multirow[t]{3}{*}{ LCX } & 0.64 & 1 & 0.80 & 0.36 & 0.75 & 0.38 & 0.65 \\
\hline & & 27 & 0.69 & 0.56 & 0.79 & 0.41 & 0.59 \\
\hline & & 57 & 0.57 & 0.68 & 0.81 & 0.38 & 0.59 \\
\hline \multicolumn{8}{|c|}{$>70 \%$ stenosis } \\
\hline \multirow[t]{3}{*}{ LM } & 0.61 & 1 & 0.40 & 0.82 & 0.27 & 0.87 & 0.75 \\
\hline & & 69 & 0.20 & 0.95 & 0.40 & 0.86 & 0.83 \\
\hline & & 105 & 0.19 & 0.97 & 0.51 & 0.86 & 0.84 \\
\hline \multirow[t]{3}{*}{ LAD } & 0.63 & 1 & 0.93 & 0.18 & 0.94 & 0.12 & 0.88 \\
\hline & & 55 & 0.84 & 0.38 & 0.95 & 0.12 & 0.81 \\
\hline & & 127 & 0.71 & 0.49 & 0.95 & 0.09 & 0.69 \\
\hline RCA & 0.67 & 1 & 0.89 & 0.31 & 0.80 & 0.90 & 0.80 \\
\hline & & 56 & 0.65 & 0.62 & 0.84 & 0.36 & 0.64 \\
\hline & & 25 & 0.76 & 0.51 & 0.82 & 0.40 & 0.69 \\
\hline LCX & 0.61 & 1 & 0.81 & 0.32 & 0.63 & 0.52 & 0.60 \\
\hline & & 24 & 0.71 & 0.47 & 0.66 & 0.52 & 0.61 \\
\hline & & 57 & 0.56 & 0.58 & 0.65 & 0.47 & 0.56 \\
\hline
\end{tabular}

a Abbreviations: CAD, coronary artery disease; CABG, coronary artery bypass graft; LM, left main; LAD, left anterior descending artery; RCA, right coronary artery; LCX, left circumflex artery; AUC, area under ROC curve; Sens,sensitivity; Spec, specificity; PPV, positive predictive value; NPV, negative predictive value.Cutoffs were selected to achieve optimum summation of sensitivity and specificity.

${ }^{b}$ Cutoffs were selected to achieve optimum summation of sensitivity and specificity. 


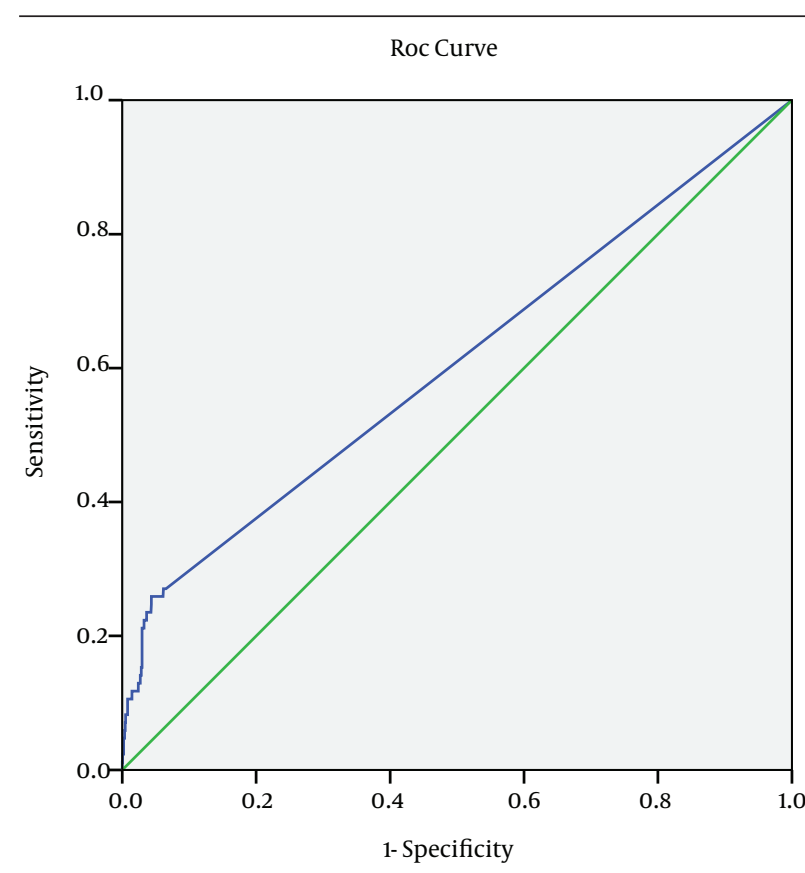

Figure 1. ROC Curve for Differentiation of Significant LM Stenoses [More Than 50\% Stenosis] Based on the LM Calcium Scoring

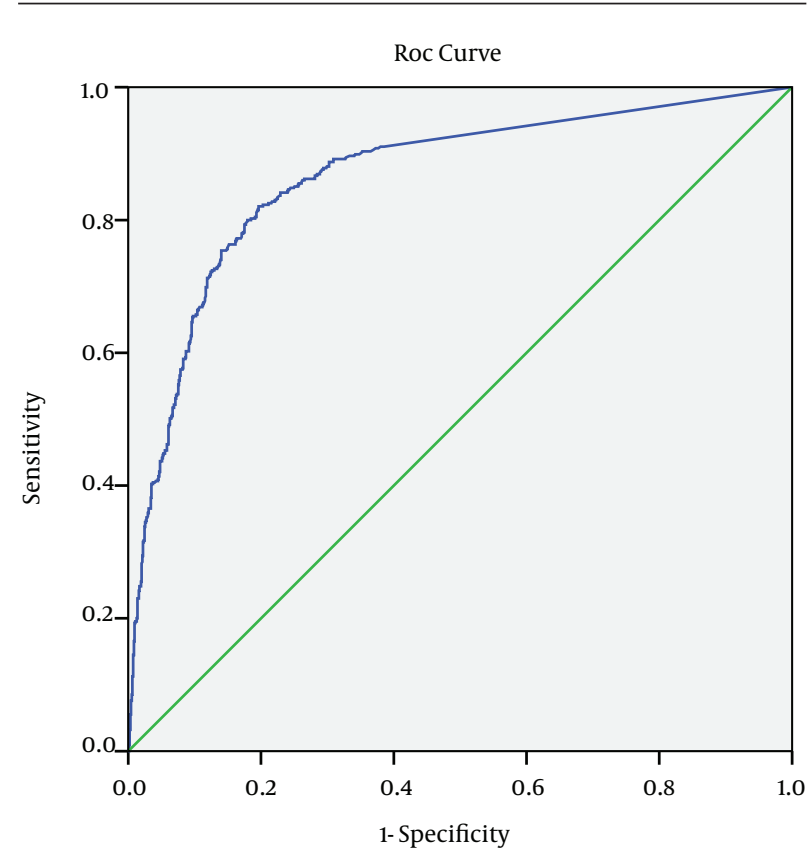

Figure 2. ROC Curve for Differentiation of Significant LAD Stenoses [More Than 70\% Stenosis] Based on the LAD Calcium Scoring

\section{Discussion}

The key finding of our study is: in stable patients without previous history of CABG or stenting, comes to hospital with the chief complaint of CAD symptoms we can perform them CAC scoring under CCTA. Our findings show

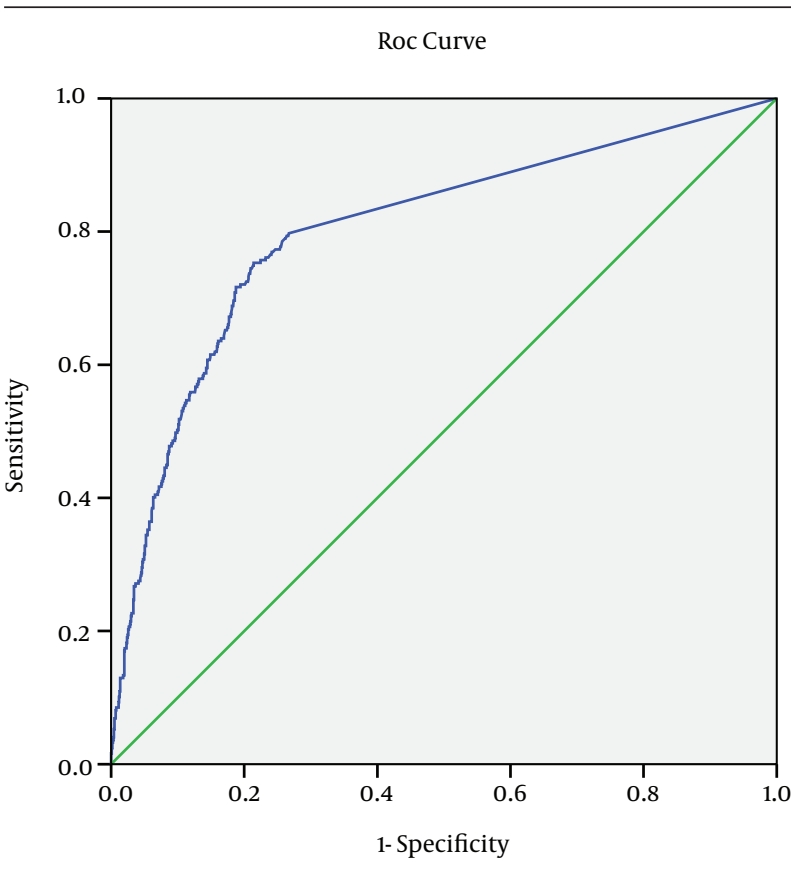

Figure 3. ROC Curve for Differentiation of Significant LCX Stenoses [More Than 70\% Stenosis] Based on the LCX Calcium Scoring

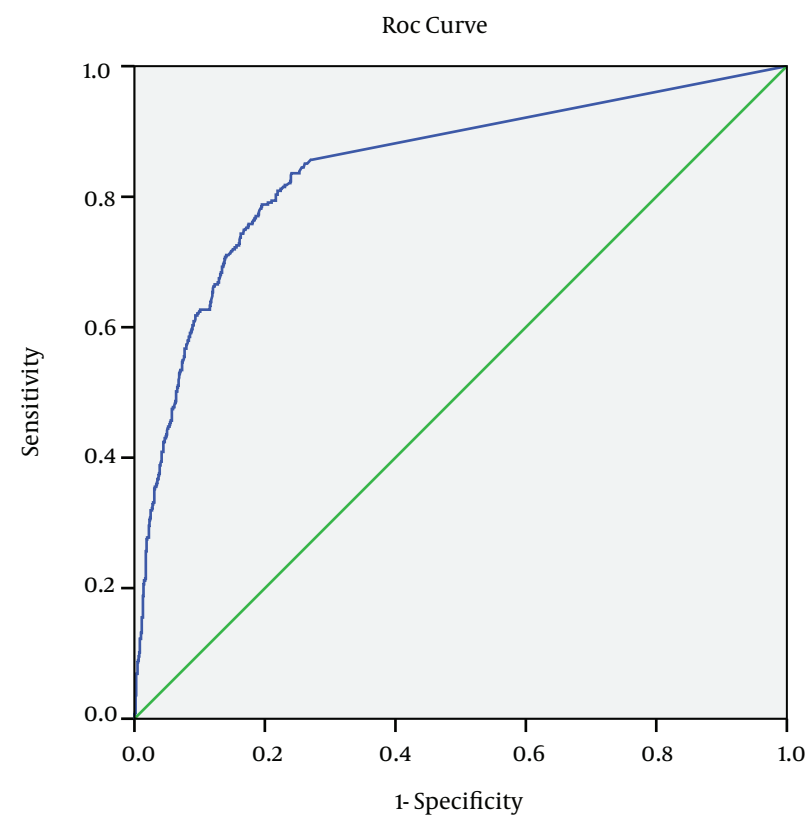

Figure 4. ROC Curve for Differentiation of Significant RCA Stenoses [More Than 70\% Stenosis] Based on the RCA Calcium Scoring

that if CAC scores findings rule out the CAD, we can rule out any obstruction $\geq 50 \%$ in coronary with more than $87 \%$ accuracy and at least $94 \%$ accuracy for obstruction $\geq$ $70 \%$ but because of low AUC and sensitivity in LM, LCX and 
RCA according to LAD, it's better use Calcium score method for ruling out obstruction in LAD. The other point here is although these values have high accuracy, considering amount of patients come to the emergency department (ED) of a hospital with the heart symptoms during one year (it accounts for approximately $8 \%$ to $10 \%$ of the 119 million ED visits yearly) (21), we would understand that a significant number of patients suffered from CAD will miss diagnosed according to CAC scoring system at the end of the year. In accordance of previous studies our study shows that although extent of CAD in CCTA are associated with extent of the CAC scores (22) and the greater grade of stenosis, the greater mean of calcium score, however; CAC score isn't sensitive enough to distinguish coronary artery obstruction (17\% sensitivity in any kind of stenosis in LM up to $81 \%$ sensitivity in $\geq 70 \%$ stenosis in LAD) and CCTA is highly superior than CAC score in CAD detection. Hadamitzky et al. (23) showed in their study that in patients with suspected CAD, CCTA can detect coronary stenosis and improves prediction of cardiac events over and above conventional risk scores and calcium scoring. Cademartiri et al. (24) concluded that CACS isinadequate for the detection of obstructive and nonobstructive CAD compared with CTCA. Striking finding of our study is that, unlike other studies, in group A that contain patients with previous history of CABG (high risk patients), particularly in LAD artery sensitivity and PPV of CAC score for CAD detection are high ( $84 \%$ and 95\% respectively), However, there is some concern that this finding is a subject to verification bias and isn't clinically meaningful. As we said previously CAC scorecan detect CAD with a high NPV (73-98\%) and have positive correlation with coronary artery stenosis grade but it's not enough to ruling out CAD. In our study in LAD we founded 947 patients with any kind of stenosis, 649 patients with stenosis $\geq 50$ and 355 patients with stenosis $\geq 70$ in which respectively we found 211 patients (21.6\%), 88 (13.5\%) and $39(10.9 \%)$ patients with zero CAC score. Rubinshtein et al. (25) showed in their study $7 \%$ patients without CAC and $17 \%$ with low CAC score in a group of patients with proven significant CAD. The Gottlieb group (26) also observed that $12 \%$ of vessels with zero CAC had significant CAD in patients with stable CAD symptoms. Thus according to our findings and previous studies CAC score doesn't have acceptable accuracy for detection of CAD and also is inadequet to ruling out coronary obstruction. while the CCTA not only detect coronary artery stenosis with a high accuracy (27) (diagnostic accuracy of CTA for detecting of > - 50\% coronary artery stenosis according to conventional angiography revealed an AUC of 0.93 (95\% confidence interval [CI]: 0.90 to 0.96 ), with a sensitivity of $85 \%$ (95\% CI: 79 to 90 ), a specificity of $90 \%$ (95\% CI: 83 to 94), a PPV of 91 \% (95\% CI: 86 to 95), and a NPV of 83 \% (95\% CI: 75 to 89)), but also is the most useful rule-out test in patients with mild coronary calcification or those with a calcium score of zero and also in patients with low to intermediate pretest probability of CAD (28). Although we and lots of oth- er studies $(23,24,29,30)$ conclude that CAC score doesn't help to diagnose or rule out CAD in symptomatic patients but we can't ignore the CAC score ability to estimate atherosclerosis in coronary artery vasculature by quantifying calcium burden in coronary artery walls (31). Budoff $M$ et al. (32) illustrated in a large observational cohort study that CAC score reveals independent information in addition to traditional risk factors in the prediction of all cardiac mortalitycauses in asymptomatic individuals referredfor CAC scanning to cardiovascular risk assessment. Detrano R and colleagues (33) performed coronary calcium scanning in a papulation without clinical cardiovascular disease and evaluate ability of CAC score in future cardiac risk stratification; they showed that coronary calcium score provides strong predictive information about future CAD incidence beyond that provide by standard risk factors. Our study have some strong points. Although coronary artery calcium score methods were evaluated in other studies, a few of them have evaluated vessel specific coronary artery calcium score prognostic value in detecting CAD. We performed the diagnostic accuracy of vessel specific CAC in detection of coronary stenosis. On the other hand our sample size is so large which increase validity of our study. Our study has some limitations. In our study gold standard for coronary stenosis is CT Angiography finding, it would be better if we could use invasive coronary angiography findings as gold standard instead, but this week point would not disturb our study results because CT angiography has acceptable sensitivity and specificity in detecting coronary stenosis. By evaluating of all these finding we can conclude that coronary calcium score provide useful and additive findings beside other cardiac risk stratification score when perform as a screening method in asymptomatic individuals but in patients with CAD symptoms who come to ED and underwent CCTA as a diagnostic method, CAC score although give useful information especially in LAD and LM, CCTA is highly superior than CAC score thus maybe we can eliminate CAC scan as a routinely perform procedure at the beginning of the CCTA and so minimize patients radiation does.

\section{References}

1. Rosamond W, Flegal K, Friday G, Furie K, Go A, Greenlund K, et al. Heart disease and stroke statistics--2007 update: a report from the American Heart Association Statistics Committee and Stroke Statistics Subcommittee. Circulation. 2007;115(5):e69-171.

2. Heidenreich PA, Trogdon JG, Khavjou OA, Butler J, Dracup K, Ezekowitz MD, et al. Forecasting the future of cardiovascular disease in the United States: a policy statement from the American Heart Association. Circulation. 2011;123(8):933-44.

3. Hosseini MP, Soltanian-Zadeh H, Akhlaghpoor S. Detection and Severity Scoring of Chronic Obstructive Pulmonary Disease Using Volumetric Analysis of Lung CT Images. Iran J Radiol. 2012;9(1):22-7.

4. Sahin H, Ceylan N, Bayraktaroglu S, Tasbakan S, Veral A, Savas R. Necrotizing Sarcoid Granulomatosis Mimicking Lung Malignancy: MDCT, PET-CT and Pathologic Findings. Iran J Radiol. 2012;9(1):37-41.

5. Shabestari AA, Akhlaghpoor S, Tayebivaljozi R, Fattahi Masrour 
F. Prevalence of Congenital Coronary Artery Anomalies and Variants in 2697 Consecutive Patients Using 64-Detector Row Coronary CTAngiography. Iran J Radiol. 2012;9(3):111-21.

6. Rezaeetalab F, Farrokh D, Zandiee B. Multiplanar Reconstructed Thoracic CT Bronchoscopy in Endobronchial Tuberculosis. Iran J Radiol.2012;9(4):234-6.

7. Iwai T, Izumi T, Inoue T, Maegawa J, Fuwa N, Mitsudo K, et al. Occipital artery arising from the anterior aspect of the internal carotid artery identified by three-dimensional computed tomography angiography. Iran J Radiol. 2012;9(2):103-5.

8. Cademartiri F, Maffei E, Palumbo A, Martini C, Seitun S, Tedeschi C, et al. Diagnostic accuracy of computed tomography coronary angiography in patients with a zero calcium score. Eur Radiol. 2010;20(1):81-7.

9. Stein PD, Yaekoub AY, Matta F, Sostman HD. 64-slice CT for diagnosis of coronary artery disease: a systematic review. Am J Med. 2008;121(8):715-25.

10. Liew GY, Feneley MP, Worthley SG. Appropriate indications for computed tomography coronary angiography. Med J Aust. 2012;196(4):246-9.

11. Achenbach S, Marwan M, Schepis T, Pflederer T, Bruder H, Allmendinger $\mathrm{T}$, et al. High-pitch spiral acquisition: a new scan mode for coronary CT angiography. J Cardiovasc Comput Tomogr 2009;3(2):117-21.

12. Fernandez-Friera L, Garcia-Alvarez A, Guzman G, Garcia MJ. Coronary CT and the coronary calcium score, the future of ED risk stratification? Curr Cardiol Rev. 2012;8(2):86-97.

13. Fink C, Krissak R, Henzler T, Lechel U, Brix G, Takx RA, et al. Radiation dose at coronary CT angiography: second-generation dualsource CT versus single-source 64-MDCT and first-generation dual-source CT. AJR Am J Roentgenol. 2011;196(5):W550-7.

14. Stolzmann P, Leschka S, Betschart T, Desbiolles L, Flohr TG, Marincek B, et al. Radiation dose values for various coronary calcium scoring protocols in dual-source CT. Int J Cardiovasc Imaging. 2009;25(4):443-51.

15. Rumberger JA, Kaufman L. A rosetta stone for coronary calcium risk stratification: agatston, volume, and mass scores in 11,490 individuals. AJR Am J Roentgenol. 2003;181(3):743-8.

16. Greenland P, Bonow RO, Brundage BH, Budoff MJ, Eisenberg MJ, Grundy SM, et al. ACCF/AHA 2007 clinical expert consensus document on coronary artery calcium scoring by computed tomography in global cardiovascular risk assessment and in evaluation of patients with chest pain: a report of the American College of Cardiology Foundation Clinical Expert Consensus Task Force (ACCF/AHA Writing Committee to Update the 2000 Expert Consensus Document on Electron Beam Computed Tomography) developed in collaboration with the Society of Atherosclerosis Imaging and Prevention and the Society of Cardiovascular Computed Tomography. J Am Coll Cardiol. 2007;49(3):378-402.

17. National Institute for Health and Clincial Excellence.. Computed tomography (CT) scanners for cardiac imaging - Somatom Definition Flash, Aquilion One, Brilliance iCT and Discovery CT750 HD. 2011.

18. Liu Q, Qian Z, Marvasty I, Rinehart S, Voros S, Metaxas DN. Lesionspecific coronary artery calcium quantification for predicting cardiac event with multiple instance support vector machines. Med Image Comput Comput Assist Interv. 2010;13(Pt 1):484-92.

19. Raff GL, Abidov A, Achenbach S, Berman DS, Boxt LM, Budoff MJ, et al. SCCT guidelines for the interpretation and reporting of coronary computed tomographic angiography. J Cardiovasc Comput Tomogr. 2009;3(2):122-36.
20. Agatston AS, Janowitz WR, Hildner FJ, Zusmer NR, Viamonte M, Jr., Detrano R. Quantification of coronary artery calcium using ultrafast computed tomography.JAm Coll Cardiol.1990;15(4):827-32.

21. Kontos MC, Diercks DB, Kirk JD. Emergency department and of fice-based evaluation of patients with chest pain. Mayo Clin Proc. 2010;85(3):284-99.

22. Kwon SW, Kim YJ, Shim J, Sung JM, Han ME, Kang DW, et al. Coronary artery calcium scoring does not add prognostic value to standard 64-section CT angiography protocol in low-risk patients suspected of having coronary artery disease. Radiology. 2011;259(1):92-9.

23. Hadamitzky M, Distler R, Meyer T, Hein F, Kastrati A, Martinoff S, et al. Prognostic value of coronary computed tomographic angiography in comparison with calcium scoring and clinical risk scores. Circ Cardiovasc Imaging. 2011;4(1):16-23.

24. Cademartiri F, Maffei E, Palumbo A, Seitun S, Martini C, Tedeschi $\mathrm{C}$, et al. Coronary calcium score and computed tomography coronary angiography in high-risk asymptomatic subjects: assessment of diagnostic accuracy and prevalence of non-obstructive coronary artery disease. Eur Radiol. 2010;20(4):846-54.

25. Rubinshtein R, Gaspar T, Halon DA, Goldstein J, Peled N, Lewis BS. Prevalence and extent of obstructive coronary artery disease in patients with zero or low calcium score undergoing 64-slice cardiac multidetector computed tomography for evaluation of a chest pain syndrome. Am J Cardiol. 2007;99(4):472-5.

26. Gottlieb I, Miller JM, Arbab-Zadeh A, Dewey M, Clouse ME, Sara L, et al. The absence of coronary calcification does not exclude obstructive coronary artery disease or the need for revascularization in patients referred for conventional coronary angiography. JAm Coll Cardiol. 2010;55(7):627-34.

27. Miller JM, Rochitte CE, Dewey M, Arbab-Zadeh A, Niinuma H, Gottlieb I, et al. Diagnostic performance of coronary angiography by 64-row CT. N Engl J Med. 2008;359(22):2324-36.

28. Arbab-Zadeh A, Miller JM, Rochitte CE, Dewey M, Niinuma H, Gottlieb I, et al. Diagnostic accuracy of computed tomography coronary angiography according to pre-test probability of coronary artery disease and severity of coronary arterial calcification. The CORE-64 (Coronary Artery Evaluation Using 64-Row Multidetector Computed Tomography Angiography) International Multicenter Study. J Am Coll Cardiol. 2012;59(4):379-87.

29. Ma ES, Yang ZG, Li Y, Dong ZH, Zhang L, Qian LL. Correlation of calcium measurement with low dose 64-slice CT and angiographic stenosis in patients with suspected coronary artery disease. Int $J$ Cardiol. 2010;140(2):249-52.

30. van Werkhoven JM, Schuijf JD, Gaemperli O, Jukema JW, Kroft LJ, Boersma E, et al. Incremental prognostic value of multi-slice computed tomography coronary angiography over coronary artery calcium scoring in patients with suspected coronary artery disease. Eur Heart J. 2009;30(21):2622-9.

31. Guerci AD, Spadaro LA, Goodman KJ, Lledo-Perez A, Newstein D, Lerner G, et al. Comparison of electron beam computed tomography scanning and conventional risk factor assessment for the prediction of angiographic coronary artery disease. J Am Coll Cardiol. 1998;32(3):673-9.

32. Budoff MJ, Shaw LJ, Liu ST, Weinstein SR, Mosler TP, Tseng PH, et al. Long-term prognosis associated with coronary calcification: observations from a registry of 25,253 patients. J Am Coll Cardiol. 2007;49(18):1860-70.

33. Detrano R, Guerci AD, Carr JJ, Bild DE, Burke G, Folsom AR, et al. Coronary calcium as a predictor of coronary events in four racial or ethnic groups. N Engl J Med.2008;358(13):1336-45. 